\title{
Visual Cerebral Microbleed Detection on $7 T$ MR Imaging: Reliability and Effects of Image Processing
}

\author{
J. de Bresser, M. Brundel, M.M. Conijn, J.J. van Dillen, M.I. Geerlings, M.A. Viergever, P.R. Luijten, G.J. Biessels
}

\begin{abstract}
SUMMARY: MR imaging at 7T has a high sensitivity for cerebral microbleed detection. We identified mIP processing conditions with an optimal balance between the number of visually detected microbleeds and the number of sections on 7T MR imaging. Even with optimal mIP processing, the limited size of some of the microbleeds and the susceptibility effects of other adjacent structures were a challenge for visual detection, which led to a modest inter-rater agreement, mainly due to missed microbleeds. Automated lesion-detection techniques may be required to optimally benefit from the increased spatial resolution offered by 7T MR imaging.
\end{abstract}

ABBREVIATIONS: $I C C=$ intraclass correlation coefficient; $M A R S=$ Microbleed Anatomical Rating Scale; $m I P=$ minimum intensity projection; $\mathrm{SI}=$ similarity index; SMART = Second Manifestations of ARTerial disease

$\mathbf{T}$ here is a growing interest in cerebral microbleeds on MR imaging in the context of aging, cerebrovascular disease, and dementia. Recent studies indicate that microbleeds may be markers of specific underlying microvascular pathologies. Furthermore, the presence of microbleeds may convey prognostic information in patients with stroke or dementia. Improvement in the detection of microbleeds is important to further our understanding of this novel marker of cerebral small-vessel disease.

The sensitivity to detect microbleeds increases with higher MR imaging field strengths. ${ }^{1}$ On $7 \mathrm{~T}$ dual-echo T2*-weighted MR imaging, for example, up to 3 times as many microbleeds can be detected than on 1.5T MR images. ${ }^{2}$ However, susceptibility effects of other structures also complicate microbleed detection at higher field strengths. ${ }^{1}$ We sought to determine mIP processing conditions with an optimal balance between accuracy and rating time and to determine intra- and interrater agreement for these processing conditions on $7 \mathrm{~T} \mathrm{MR}$ imaging.

Received April 5, 2011; accepted after revision October 25.

From the Image Sciences Institute (J.d.B., M.A.V., P.R.L.); Department of Neurology (J.d.B., M.B., J.J.v.D., G.J.B.), Rudolf Magnus Institute of Neuroscience; Department of Radiology (M.M.C., P.R.L.); and Julius Center for Health Sciences and Primary Care (M.M.C., M.I.G.), University Medical Center Utrecht, Utrecht, the Netherlands. This work was supported by a High Potential grant from Utrecht University.

Please address correspondence to Jeroen de Bresser, Department of Neurology, University Medical Center Utrecht, G03.228, PO Box 85500, Heidelberglaan 100, 3508 GA Utrecht, the Netherlands; e-mail: J.deBresser@umcutrecht.nl

http://dx.doi.org/10.3174/ajnr.A2960

\section{TECHNIQUE}

\section{Subjects}

Subjects were selected from the SMART 7T MR imaging study. ${ }^{2}$ The SMART study is a prospective cohort study that assesses brain changes in patients with manifest arterial disease. For the comparison of the different mIP conditions, we first selected scans from 3 subjects (mean age, $72 \pm 8$ years) who each had at least 10 microbleeds on their 7T MR images, based on previous evaluations by raters who were not involved in the present study.

Subsequently, scans from 10 additional subjects ( 8 with and 2 without microbleeds; mean age, $67 \pm 8$ years; the raters were not aware of the number of subjects with and without microbleeds) were selected to study intra- and inter-rater agreement. This study was approved by the local ethics committee of the University Medical Center Utrecht, and all subjects signed an informed consent form.

\section{MR Imaging Scanning Protocol}

Scans were acquired on a 7T MR system (Philips Healthcare, Cleveland, Ohio) with a volume-transmit and 16-channel receive head coil (Nova Medical, Wilmington, Massachusetts). Dualecho 3D T2*-weighted images were obtained (TR/first TE/second $\mathrm{TE}=20 / 2.5 / 15 \mathrm{~ms}$ ), with an acquired voxel size of $0.4 \times 0.4 \times 0.6$ $\mathrm{mm}^{3}$, reconstructed to $0.35 \times 0.35 \times 0.3 \mathrm{~mm}^{3}$ (for details see Conijn et $\left.\mathrm{al}^{1}\right)$. FLAIR images were obtained (TR/TE/TI $=8000 /$ $294 / 2315 \mathrm{~ms}$ ) with an acquired voxel size of $0.99 \times 1.00 \times 1.10$ $\mathrm{mm}^{3}$, reconstructed to $0.98 \times 0.98 \times 0.55 \mathrm{~mm}^{3}$.

Eight of the 10 subjects also had a 1.5T MR imaging scan (Gyroscan ACS-NT; Philips Healthcare, Best, the Netherlands) with 
$3 \mathrm{D} \mathrm{T} 2^{*}$-weighted images $(\mathrm{TR} / \mathrm{TE}=25 / 35 \mathrm{~ms}$ ) with an acquired voxel size of $0.8 \times 0.8 \times 0.8 \mathrm{~mm}^{3}$ (for details see Conijn et $\mathrm{al}^{2}$ ) and T2-weighted images $(\mathrm{TR} / \mathrm{TE}=2200 / 100 \mathrm{~ms})$ with an acquired voxel size of $0.90 \times 1.03 \times 4.00 \mathrm{~mm}^{3}$ on the same day as the $7 \mathrm{~T}$ scan.

\section{Image Processing and Design of the Study}

We first determined the optimal mIP conditions for visual microbleed rating at 7T. Scans of the 3 subjects with multiple microbleeds were processed with variations in section thickness (section thickness/gap [negative gap means overlap] in millimeters: $1 / 0,2 / 0,3 / 0,10 / 0)$ and overlap $(3 /-2,5 /-4,4 /-2,10 /-8)$ and rated by 1 author (J.J.v.D.).

Because the 2/0 and 4/-2 mIP condition were found to have an optimal tradeoff between the number of detected microbleeds and the number of sections, these conditions were selected to assess intra- and inter-rater agreement and the effect of overlap. In this stage of the study, 7T scans of 10 subjects were rated by 4 authors (J.d.B., M.B., J.J.v.D., G.J.B), 1 of whom rated all scans twice (J.J.v.D.).

The $1.5 \mathrm{~T}$ scans were processed with 5/0 mIP, in line with previous studies (eg, Gregoire et $\mathrm{al}^{3}$ ). These scans were also rated by the 4 authors (J.d.B., M.B., J.J.v.D., G.J.B), 1 of whom rated all scans twice (J.J.v.D.).

\section{Microbleed Rating}

For the 7T scans, microbleeds were rated with concomitant onscreen presentation of the first- and second-echo mIP processed images. Ratings were performed by the MARS. ${ }^{3}$ Microbleeds were defined as black, round, or ovoid lesions on the first- or secondecho image and distinguished into possible or definite microbleeds. ${ }^{3}$ Mimics, like vessels and symmetric calcifications in the basal ganglia and choroid plexus, were excluded. ${ }^{3}$ FLAIR or T2weighted images were used to detect infarcts. Microbleeds were not rated within infarcted brain tissue. We made 2 modifications to the MARS for application on the 7T scans. First, we used no lower size limit for the lesions ( $2 \mathrm{~mm}$ is the lower size limit of MARS). Second, lesions visible on the first-echo image had to be larger on the second-echo image (so-called blooming) to be rated as microbleeds. ${ }^{2}$ Before the formal rating, the raters practiced the rating method together on $7 \mathrm{~T}$ scans of individuals from the SMART study who were not included in the present study. Levels of experience with brain MR imaging rating varied across raters. Nevertheless, on the $1.5 \mathrm{~T}$ scans intra- and inter-rater agreement was excellent (intrarater: number of definite [ICC, 0.89] and total microbleeds [0.91]; inter-rater agreement of all raters: number of definite [0.95] and total microbleeds [0.91]).

\section{Ground Truth Ratings}

To assess the effect of mIP with an overlap, we saved the precise spatial locations of all identified definite microbleeds on 7T scans of the 10 subjects and used them to establish "ground truth" ratings. Each subject was rated 8 times (ie, for 2 conditions of mIP processing by 4 raters). A lesion on a particular location was labeled as a definite microbleed for the ground truth rating if it had been labeled as such on at least 5 of the 8 ratings. Lesion locations that had been marked as definite microbleeds on 4 or fewer of the
8 ratings were examined in a consensus meeting with 3 raters (J.d.B., M.B., G.J.B.) and were included or excluded from the ground truth rating on the basis of the majority of votes.

\section{Statistical Analysis}

Intra- and inter-rater agreement of the number of rated microbleeds (possible, definite, and total [possible + definite]) were calculated as an ICC for the $2 / 0$ and $4 /-2$ mIP processed scans of the 10 subjects.

The effect of an overlap in the mIP processing of 7T scans was assessed by comparing $2 / 0$ with $4 /-2 \mathrm{mIP}$ processing within the 10 subjects. Individual definite microbleed locations were compared between rated scans of each rater with the ground truth ratings to determine true-positives, false-positives, and false-negatives. These values were used to calculate a sensitivity and SI. The sensitivity and SI were compared between $2 / 0$ and $4 /-2$ processing.

\section{RESULTS}

\section{Different Conditions of mIP Processing}

On the scans of the 3 subjects that were used to determine optimal $\mathrm{mIP}$ processing conditions, the maximum numbers of detected total microbleeds per subject were 11,15 , and 188 . In scans with nonoverlap mIP processing, the proportion of detected microbleeds decreased with increasing section thickness (Fig 1; percentage of maximum detected number of microbleeds averaged for the 3 subjects: $1 / 0=91 \%, 2 / 0=82 \%, 3 / 0=74 \%, 10 / 0=56 \%$ ). This effect of section thickness was less marked in mIP-processed scans with overlap $(3 /-2=95 \%, 5 /-4=96 \%, 4 /-2=80 \%$, $10 /-8=80 \%$ ). mIP processing with $1 / 0,3 /-2$, and $5 /-4$ resulted in a total of 100 sections; $2 / 0,4 /-2$, and $10 /-8$ in 50 sections; $3 / 0$ in 33 sections; and 10/0 in 10 sections. More sections increased the rating time: A total of 100 sections resulted in 20-80 minutes rating time per scan; 50 sections, in $20-47$ minutes; 33 sections, in 23-35 minutes; and 10 sections, in 7-60 minutes. On the basis of the number of detected microbleeds and number of sections, $2 / 0$ and $4 /-2 \mathrm{mIP}$ processing were selected for the second stage of the study.

\section{Intra- and Inter-Rater Agreement}

Figure 2 illustrates some of the potential difficulties of microbleed detection on 7T MR imaging.

The intrarater agreement for rating the number of microbleeds on $7 \mathrm{~T}$ scans was good for definite (ICC, mean of $2 / 0$ and 4/-2: 0.73 ) and total microbleeds (0.74), but poor for possible microbleeds (0.33). The inter-rater agreement for rating the number of microbleeds on $7 \mathrm{~T}$ scans was moderate for definite microbleeds (0.46), lower for total microbleeds (0.38), and poor for possible microbleeds (0.06).

\section{Effect of Using an Overlap in mIP Processing}

The ground truth ratings of the 10 subjects included a median number of 3 definite microbleeds (range, $0-7$ ), with a total of 36 definite microbleeds, of which 31 were lobar, 4 were deep, and 1 was cerebellar. The Table presents the ratings of individual definite microbleed locations per rater compared with the ground truth rating. Mean rating times of all raters between scans with 2/0 

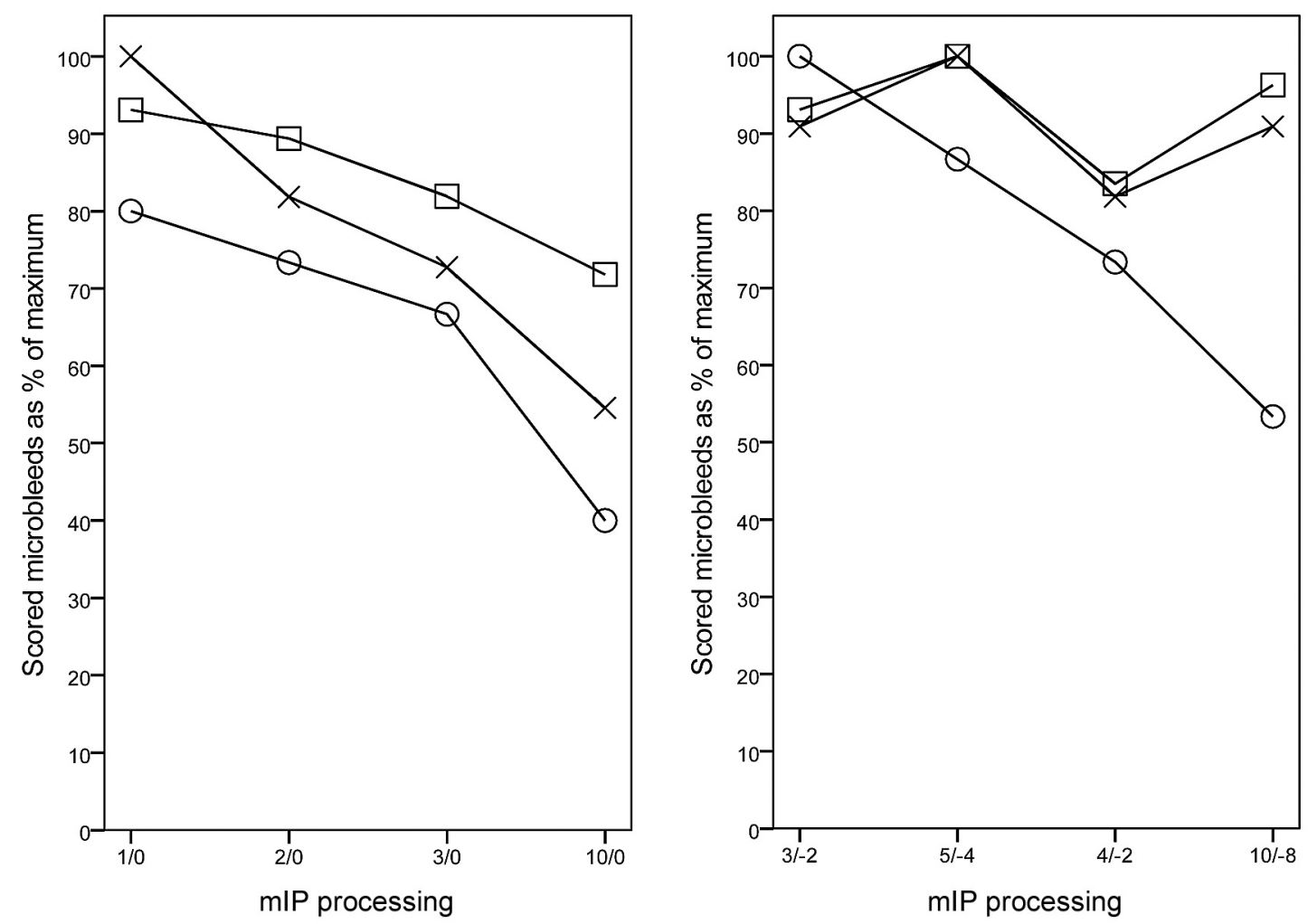

Subject 1

$\square$ Subject 2

$\times$ Subject 3

FIG 1. Total number of microbleeds for mIP processing with and without an overlap. The left figure shows results of mIP processing without overlap but with differences in section thickness. The right figure shows the results of processing with overlap. The total number of detected microbleeds (possible and definite) is shown for all subjects, expressed as a percentage of maximum detected microbleeds within subjects.

Visual rating of individual definite microbleed locations compared with the ground truth rating ${ }^{\mathrm{a}}$

\begin{tabular}{|c|c|c|c|c|c|c|c|c|c|c|}
\hline & \multicolumn{5}{|c|}{ 2/0 mIP Processing } & \multicolumn{5}{|c|}{ 4/-2 mIP Processing } \\
\hline & Rater 1 & Rater 2 & Rater 3 & Rater 4 & $\begin{array}{c}\text { Mean of } \\
\text { All Raters }\end{array}$ & Rater 1 & Rater 2 & Rater 3 & Rater 4 & $\begin{array}{l}\text { Mean of } \\
\text { All Raters }\end{array}$ \\
\hline Mean time per subject (min) & 42 & 18 & 24 & 35 & 30 & 42 & 16 & 23 & 34 & 29 \\
\hline Lobar (sensitivity/SI) & $0.61 / 0.63$ & $0.23 / 0.37$ & $0.61 / 0.72$ & $0.71 / 0.76$ & $0.54 / 0.62$ & $0.65 / 0.73$ & $0.42 / 0.59$ & $0.52 / 0.65$ & $0.81 / 0.83$ & $0.60 / 0.70$ \\
\hline Deep (sensitivity/SI) & $1.00 / 0.89$ & $0.75 / 0.75$ & $0.75 / 0.55$ & $0.75 / 0.40$ & $0.81 / 0.65$ & $0.50 / 0.36$ & $0.75 / 0.75$ & $0.50 / 0.67$ & $1.00 / 0.46$ & $0.69 / 0.56$ \\
\hline Total (sensitivity/SI) & $0.67 / 0.68$ & $0.31 / 0.46$ & $0.64 / 0.70$ & $0.72 / 0.69$ & $0.58 / 0.63$ & $0.61 / 0.66$ & $0.44 / 0.60$ & $0.53 / 0.67$ & $0.83 / 0.77$ & $0.60 / 0.67$ \\
\hline
\end{tabular}

${ }^{a}$ Ratings for each rater, which consisted of individual definite microbleed locations on scans with $2 / 0$ and $4 /-2$ mIP processing, were compared with the ground truth ratings by sensitivity and SI.

and 4/-2 mIP processing were comparable (29 minutes per scan on average). For individual definite microbleed locations for each rater compared with the locations in the ground truth ratings, 4/-2 mIP (mean sensitivity/SI of all raters, 0.60/0.67) had a slightly higher sensitivity and SI than $2 / 0 \mathrm{mIP}(0.58 / 0.63)$. Microbleed localization (lobar or deep) only had a limited effect on the reliability of visual detection.

\section{DISCUSSION}

Improvement in the detection of microbleeds is important to further our understanding of this novel marker of cerebral smallvessel disease. With 7T dual-echo $\mathrm{T} 2^{*}$-weighted MR imaging smaller and up to 3 times as many microbleeds can be detected than on $1.5 \mathrm{~T}$ MR imaging. ${ }^{2}$

In contrast to lower resolution scans, at 7T, MR image processing by means of mIP is required to aid the visual rating and reduce the large number of sections. ${ }^{2}$ We showed that scans with mIP processing of $2 / 0$ and $4 /-2$ showed an optimal balance be- tween the number of detected microbleeds and the number of sections and thus the rating time. Furthermore, detection of microbleeds could benefit from mIP processing with an overlap. Other factors (ie, pulse sequence, sequence parameters, spatial resolution, and the use of susceptibility-weighted imaging) could also influence the reliability of microbleed detection ${ }^{4}$ and should be standardized within studies and even between studies to allow comparison of results.

Even under optimal mIP conditions, inter-rater agreement of the visual rating of microbleeds on 7T MR imaging was only moderate and lower than the inter-rater agreement for the same raters at $1.5 \mathrm{~T}$. The major source of disagreement between raters was that raters missed microbleeds. This is probably due to the limited size of some of these lesions, susceptibility effects of other structures, ${ }^{1,4}$ and the large number of sections for every subject. Disagreement was more marked for possible than for definite microbleeds, and the rating of possible microbleeds on $7 \mathrm{~T}$ scans proved 


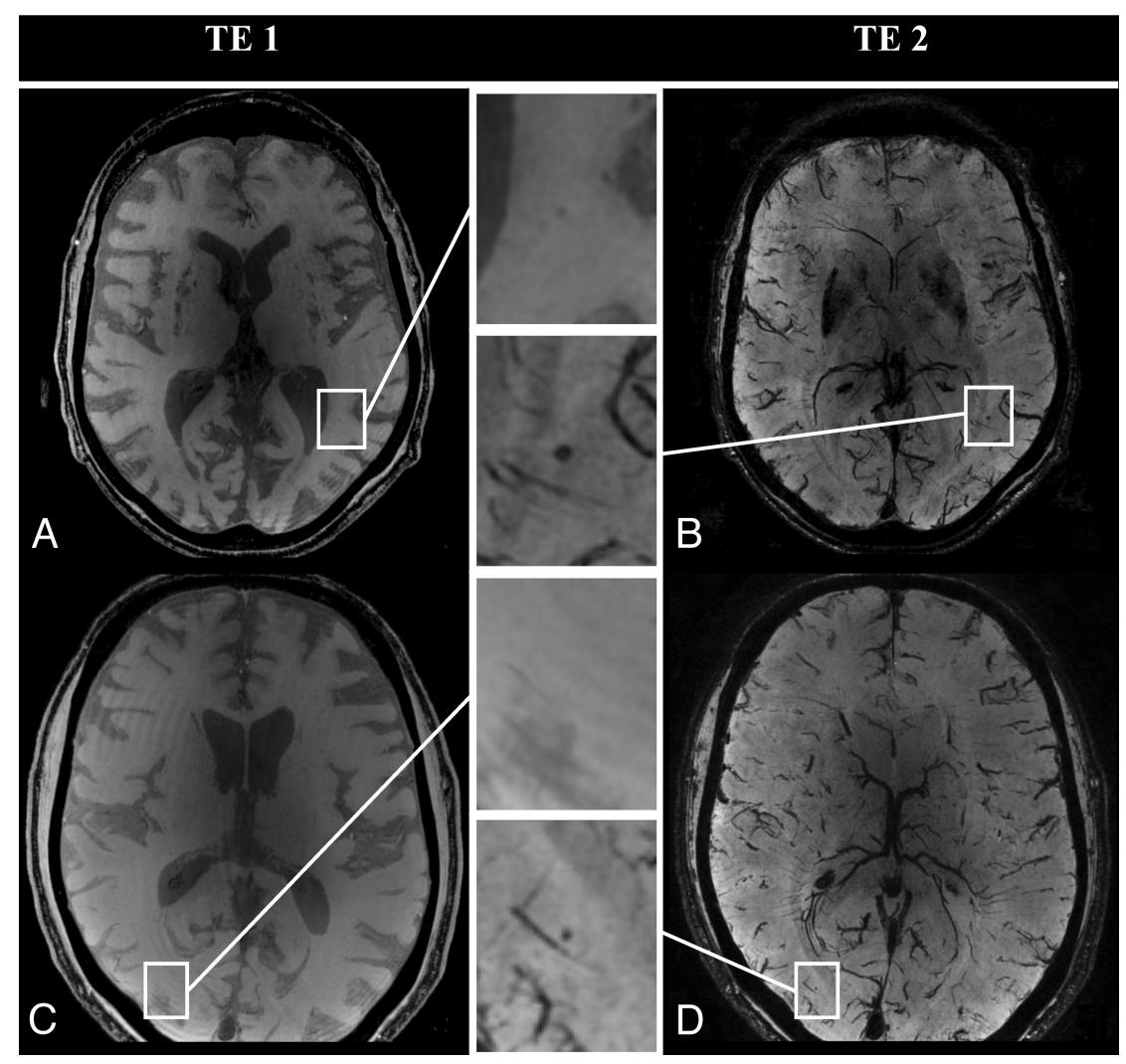

FIG 2. Examples of microbleeds that are easy or hard to detect. This figure illustrates different microbleeds that were classified as definite microbleeds in the ground truth ratings. $A$ and $B$, The TE 1 and TE 2 of the T2*-weighted 7T MR scan with a microbleed that was detected by all raters. The microbleed is seen on both the TE 1 and TE 2 images. $C$ and $D, A$ microbleed that was only detected by a few raters. This microbleed was only seen on the TE 2 image.

to be unreliable. The accuracy of the rating of the $1.5 \mathrm{~T}$ scans fell well within the range of previous studies (eg, Gregoire et $\mathrm{al}^{3}$ ), indicating that the disagreement was attributable to the properties of the $7 \mathrm{~T}$ scans rather than to poor performance of the raters.

\section{CONCLUSIONS}

7T MR imaging improves cerebral microbleed detection and will further our understanding of the pathophysiology of these lesions. Visual rating of microbleeds at 7T MR imaging should preferably involve multiple trained raters to ensure optimal lesion detection. At present, microbleed detection at 7T MR imaging should, in our view, be primarily used in a research setting. Further studies are needed to establish the clinical relevance of microbleeds on 7T MR imaging. Moreover, rating times need to be reduced to ensure further dissemination of the technique. This could be achieved by automated or semiautomated image analysis methods, which are currently becoming available. ${ }^{5}$
Disclosures: Jeroen de Bresser—RELATED: Grant: High Potential grant from Utrecht University. Max A. Viergever-UNRELATED: Grants/Grants Pending: Philips Healthcare. Geert Jan Biessels—RELATED: Grant: Utrecht University, ${ }^{*}$ Comments: unrestricted grant for my research program. (*Money paid to the institution.)

\section{REFERENCES}

1. Conijn MM, Geerlings MI, Luijten PR, et al. Visualization of cerebral microbleeds with dual-echo $\mathrm{T} 2{ }^{*}$-weighted magnetic resonance imaging at 7.0 T. J Magn Reson Imaging 2010;32:52-59

2. Conijn MM, Geerlings MI, Biessels GJ, et al. Cerebral microbleeds on MR imaging: comparison between 1.5 and 7T. AJNR Am J Neuroradiol 2011;32:1043-49

3. Gregoire SM, Chaudhary UJ, Brown MM, et al. The Microbleed Anatomical Rating Scale (MARS): reliability of a tool to map brain microbleeds. Neurology 2009;73:1759-66

4. Greenberg SM, Vernooij MW, Cordonnier C, et al. Cerebral microbleeds: a guide to detection and interpretation. Lancet Neurol 2009;8:165-74

5. Kuijf HJ, de Bresser J, Geerlings MI, et al. Efficient detection of cerebral microbleeds on 7.0 T MR images using the radial symmetry transform. Neuroimage 2012;59:2266-73 